\title{
A perspective on scaling up quantum computation with molecular spins
}

\author{
S. Carretta, ${ }^{1,2}$ D. Zueco, ${ }^{3}$ A. Chiesa, ${ }^{1,2}$ Á. Gómez-León, ${ }^{4}$ and F. Luis ${ }^{3}$ \\ 1) Department of Mathematical, Physical and Computer Sciences, University of Parma (Italy) \\ ${ }^{2)}$ UdR Parma, INSTM, I-43124 Parma, Italy \\ 3) Instituto de Nanociencia y Materiales de Aragón (INMA), CSIC-Universidad de Zaragoza, \\ 50009 Zaragoza (Spain) \\ ${ }^{4)}$ Instituto de Física Fundamental, CSIC, 28006 Madrid (Spain)
}

(Dated: 4 May 2021)

Artificial magnetic molecules can contribute to progressing towards large scale quantum computation by: a) integrating multiple quantum resources and b) reducing the computational costs of some applications. Chemical design, guided by theoretical proposals, allows embedding nontrivial quantum functionalities in each molecular unit, which then acts as a microscopic quantum processor able to encode error protected logical qubits or to implement quantum simulations. Scaling up even further requires "wiring-up" multiple molecules. We discuss how to achieve this goal by the coupling to on-chip superconducting resonators. The potential advantages of this hybrid approach and the challenges that still lay ahead are critically reviewed.

\section{INTRODUCTION}

A crucial challenge for the development of quantum technologies is to reach a computational power able to tackle problems of social and economical value ${ }^{112}$ Estimating what is necessary depends on details of the problem itself and of the platform chosen to solve it. Yet, it appears that performing quantum simulations or primenumber factorization of relevance to applications will demand operating over many thousands, even millions of qubits. ${ }^{3}$ This daunting prediction arises not only from the complexity of such problems, but also from the need of protecting quantum operations from noise and the fact that quantum error correction (QEC) is based on increasing the number of physical qubits encoding each logical (error-protected) qubit! $\underline{45}$

Although there is hope that the already available Noisy Intermediate-Size Quantum devices (NISQs) will be useful for some specific tasks,$\frac{6}{8}$ it makes sense to consider alternatives. Electron spins in semiconductor quantum $\operatorname{dot} s^{9}$ or atomic impurities ${ }^{10}$ represent natural candidates to attain high levels of integration. Progress along this direction, although encouraging, 11,13 faces problems of qubit reproducibility similar to those encountered with other circuits fabricated by top-down lithography or of qubit tunability.

Here, we focus on a different class of spin systems, based on artificial magnetic molecules $\frac{17 / 18}{18}$ They combine a microscopic, thus close to perfectly reproducible, nature with the ability of chemically designing their properties. Each of them consists of one to a few magnetic ions stabilized and protected by a shell of organic ligand molecules (Fig. 11). Molecules with an effective $S=1 / 2$ ground state provide the simplest qubit realizations but, as it is discussed below, there exist many other appealing possibilities.

Our aim is to discuss the potential that such molecular building blocks have to progress towards largescale quantum computation and the advantages they offer for the implementation of some specific applications.
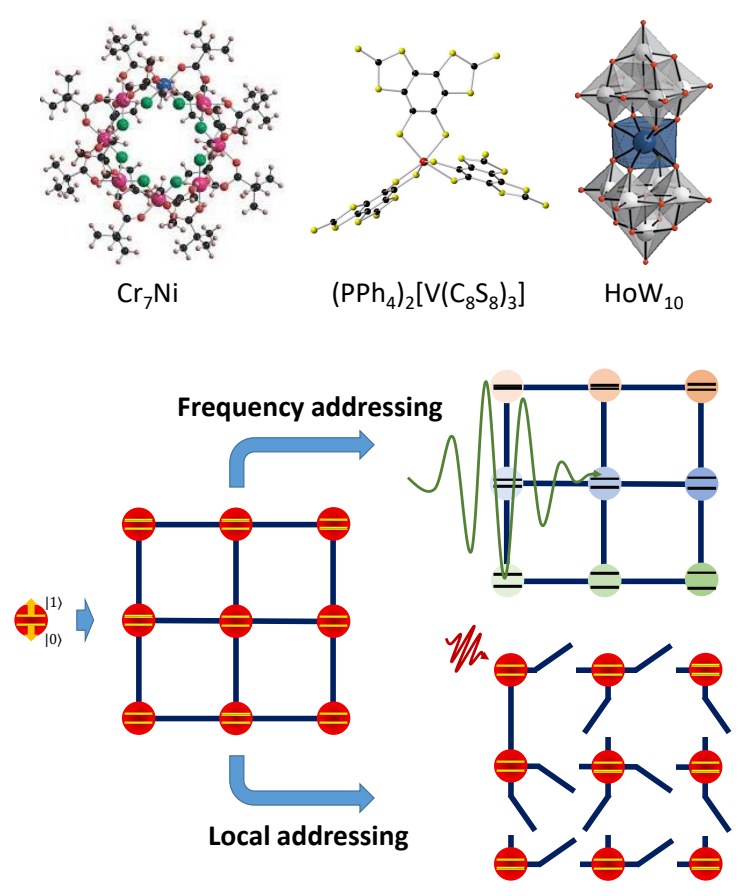

FIG. 1. (a) Molecular structures of three molecular spin qubits. In the first two, ${ }^{14 \mid 15}$ the qubit is encoded in the states of a $\operatorname{spin} S=1 / 2$. For $\mathrm{HoW}_{10} \frac{\sqrt{16}}{\text { it }}$ is defined by the tunnel split states arising from the $m= \pm 4$ total angular momentum projections. (b) Illustration of scaling up by a global frequency addressing and by a local addressing with switchable qubit-qubit interactions.

We consider two alternatives for scaling-up, which are schematically illustrated by Fig. 1. The first is based on the asymmetry between different qubits in the array (e.g. each having a different frequency) and on the interactions between them. The ensuing energy level anharmonicity then allows one to address each operation by simply choosing the adequate frequency (or "color") of a resonant electromagnetic pulse acting on the whole array. This strategy allows scaling up by "Chemistry", i.e. 
within each molecule. The second option involves a local control over each qubit and over its interactions with the rest. It relies on the very challenging goal of controlling and "wiring up" individual molecular spins.

\section{SCALING UP WITHIN EACH MOLECULE: MOLECULAR QUANTUM PROCESSORS}

A characteristic trait of molecular systems is the nearly boundless opportunities to tune their physical properties via changes in composition and structure. The molecular design allows, for instance, suppressing decoherence by either removing "magnetic noise" sources ${ }^{14|15| 19 \mid 23}$ (e.g. replacing some of the molecules in the crystal by nonmagnetic derivatives, dissolving them in adequate solvents or reducing the number of nuclear spins) or by encoding the qubit states in "decoherence-free" subspaces formed near level anticrossings $\frac{16 \mid 24}{26}$ The application of these methods has led to very significant improvements in spin coherence times $T_{2}$ which for some examples are near one ms $\frac{15}{15}$

Chemical design can also be exploited to expand the available computational space from single qubits to $d$-dimensional qudits at the level of a microscopic physical object. An option is to create molecular structures hosting several magnetic centres ${ }^{27}$ Examples include molecular dimers and trimers of lanthanide ions, $\frac{28 \mid 30}{2}$ as well as supramolecular structures able to bind several well-known molecular qubits, such as $\left[\mathrm{Cr}_{7} \mathrm{Ni}\right]$, and combine them with other $S=1 / 2$ complexes. 31

An alternative is to exploit internal spin degrees of freedom. For instance, the electronic spin $S=7 / 2$ of a $\mathrm{Gd}^{3+}$ ion defines $2^{3}=8$ discrete levels. In a well-chosen molecular coordination (see Fig. 2), leading to a sufficiently weak magnetic anisotropy and correspondingly small level splittings, these states can encode a $d=8$ qudit or 3 qubits ${ }^{[34}$ It is also possible to make use of the metal ions' nuclear spin states. ${ }^{35 / 36}$ The hyperfine coupling to the electronic spin splits these levels and considerably speeds up the rates at which such states can be coherently manipulated by electromagnetic pulses $37+39$ The different strategies can also be combined to further increase the qudit dimension. For instance, molecular structures with several magnetic ions, each acting as a qudit, can be synthesized. An illustrative example of a Gd dimer $\left(2^{6}=64\right.$ levels or 6 qubits $)$ is shown in Fig. 2 [40

The crucial question is then whether one of these molecular qudits is able to implement any quantum algorithm. ${ }^{6 / 41}$ Even though the physical operation principles are quite similar, magnetic molecules have a crucial advantage over NMR quantum computing with organic molecules ${ }^{42}$ The sizeable energy splitting between the ground and excited levels, larger than $400 \mathrm{MHz}$ even for hyperfine split levels at low magnetic fields, allows initialization of the spin state by cooling to experimentally attainable temperatures $\lesssim 10 \mathrm{mK}$. Checking universal-



(b)
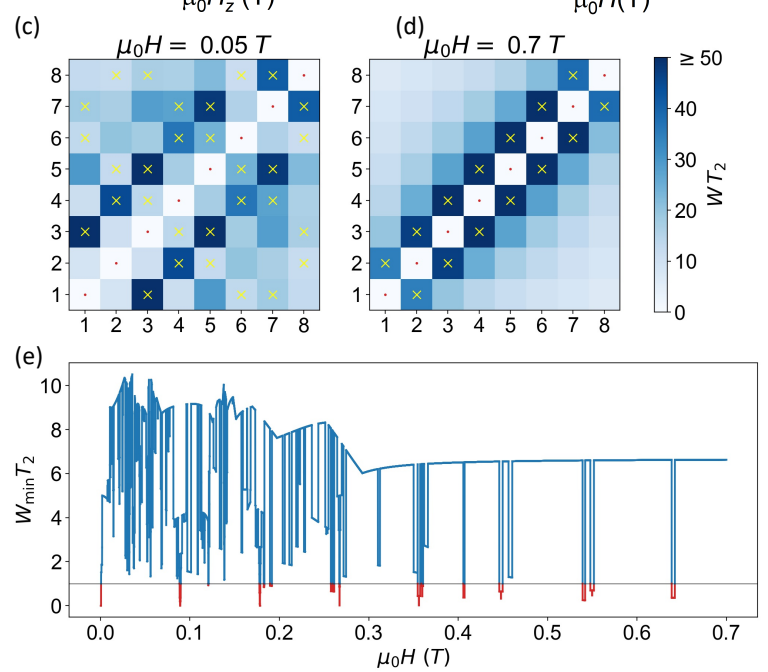

FIG. 2. Universality conditions for molecular spin qudits. (a) and (b), energy levels of two molecular spin qudits based on the $S=7 / 2$ states of $\mathrm{Gd}^{3+}$ ions: $\mathrm{GdW}_{30}$ with dimension $2^{3}=8$ and $\left[\mathrm{Gd}_{2}\right]$ with dimension $2^{6}=644^{34 \mid 40}$ Their structures are shown as insets. (c) and (d), rates $W_{n, m}$ of quantum operations, performed by sequences of resonant electromagnetic pulses, linking basis states $n$ and $m$ of $\mathrm{GdW}_{30}$ at two different magnetic fields. The yellow crosses mark the direct resonant transitions that have a Rabi frequency $\Omega_{\mathrm{R}}>1 / T_{2}$, with $T_{2} \simeq 1 \mu$ s the spin coherence time. The red dots mark the trivial identity operation. (e) Universality parameter $W_{\min } T_{2}$ for $\mathrm{GdW}_{30}$ as a function of magnetic field, where $W_{\min }$ is the smallest operation rate between any pair of basis states. The red traces signal magnetic fields where the system becomes non-universal as a result of accidental degeneracies between two non-forbidden resonant transitions.

ity then reduces itself to showing that any gate operation connecting any two arbitrary states can be realized within the spin decoherence time $\mathrm{T}_{2} \cdot \frac{34|40| 43]}{45}$ The situation is illustrated in Fig. 2 $\mathrm{c}$ c and $\mathrm{d}$ for a $d=8$ $\mathrm{GdW}_{30}$ qudit. The plots show the rates $W_{n, m}$ of operations linking basis states $n$ and $m$, implemented by sequences of resonant transitions. The Rabi frequency $\Omega_{\mathrm{R}}$ sets the frequency uncertainty of a finite duration $\left(\sim 1 / \Omega_{\mathrm{R}}\right)$ pulse. Addressability can then be enforced by choosing only those transitions whose resonant frequencies fulfill $\omega_{j}-\omega_{i}>\Omega_{\mathrm{R}}$, for any $i$ and $j$. This level anharmonicity also allows reading out the spin states $\underline{46}, 48$ A molecular spin qudit is universal when all $W_{n, m} T_{2}>1$. The lowest $W_{n, m} T_{2}$ (Fig. 2 (e)) allows benchmarking the 
performances of different molecules or of molecules with respect to other schemes ${ }^{39}$

For a given molecular qudit, $W_{n, m}$ depend on the relative strengths of the Zeeman interaction, magnetic anisotropy and hyperfine couplings. Under carefully chosen conditions, it is possible to combine universal operation with a large number of direct and fast links between pairs of states (compare panels (c) and (d) in Fig. 2). This possibility can reduce the number of operations required to implement certain gates and algorithms and thus help molecular NISQs to reach higher "quantum volumes ${ }^{1 / 8}$ than platforms based on linking nearest neighbour qubits. However, as discussed in Sec. IV, the number of levels within a molecular processor cannot be increased at will. At some point, actual scalability of the proposed platform requires to wire-up different molecular units by coupling them to resonant cavities.

\section{QUANTUM ERROR CORRECTION AND QUANTUM SIMULATIONS WITH MOLECULAR SPIN QUDITS}

The fundamental point behind $Q E C$ algorithms is to exploit a Hilbert space with dimension larger than 2 to encode a logical qubit. This extra space can make errors detectable and correctable ${ }^{45}$ Furthermore, when considering a specific physical implementation of qubits in the NISQ era, $\frac{6}{6}$ another important issue is to design QEC schemes correcting the most important errors occurring in the real hardware. Here, the interaction with neighboring nuclear spins leads to the decay of out-of-diagonal elements of the density matrix of the central spins, thus destroying the quantum computation. Hence, we focus on QEC codes designed to counteract dephasing and we discuss how molecular nanomagnets can be exploited to define qubits with embedded QEC [30|38/49

Two routes have been put forward to embed QEC in single magnetic molecules. On the one hand, standard block codes can be efficiently implemented in molecules made of weakly interacting spins $1 / 2 \cdot 30$ On the other, effective qudit QEC codes can be implemented exploiting the $d=2 S+1$ levels of a spin $S$ system.49151/52

The first approach was investigated in Ref. 30, where it was shown that an [ErCeEr] trimer is a promising molecule to encode a logical qubit protected against dephasing by the three-qubit phase-flip code. This implementation requires three weakly interacting (effective) spin $1 / 2$, in order to avoid the occurrence of correlated errors which are not handled by this code. However, the interaction between the qubits must be sufficient to enable excitations of one of the spin conditioned to the state of the others. Moreover, significantly different $g$ values enable the use of fast manipulation pulses, which are crucial because dephasing acts also during the implementation of QEC. Rare-earth Kramers ions such as Er and Ce perfectly fit these requirements. $\cdot 30$

The second approach was proposed in Ref. 49. It exploits a single electronic or nuclear spin qudit $S$ to en- code an error-protected qubit. This is done by designing code words (consisting of superpositions of qudit states) which are robust against error-operators characterizing the incoherent dynamics of the central spin. Specialization to real errors gives a substantially better performance compared to abstract generic error models 5 In the simplest modelling of the bath, error operators are derived from a perturbative expansion of the solution of the Lindblad equation, ${ }^{49}$ but the derivation of optimized code words can be extended to more realistic nuclear bath dynamics $\underline{52}$

Once the code words have been determined, a proper sequence of electromagnetic pulses can be designed to implement the QEC code on a given molecular hardware (see Fig. 3. $(a, b))$. The simplest physical realization is represented by a nuclear spin $S$ qudit coupled to a spin $1 / 2$ electronic ancilla, used to detect errors. The gain is remarkable already for a minimal $S=3 / 2$ qudit, realized e.g. in $\left(\mathrm{PPh}_{4}\right)_{2}\left[\mathrm{Cu}(\mathrm{mnt})_{2}\right]$ complex ${ }^{21}$ (Fig. 3-(a)). It shows a maximum at intermediate $T / T_{2}$ (Fig. 3f(c)), due to the finite duration of the QEC procedure included in the simulation, which bounds the error for small $T / T_{2}$. Being effective also for long memory times, the scheme allows many gates to be implemented before error correction is needed, which could constitute a very important advantage in the NISQ era.

Both approaches outlined above are able to defeat pure dephasing already in the two minimal implementations, represented by 3 spins $1 / 2$ or by a spin $3 / 2$ qudit 30149 . The latter however appears simpler (a single spin) and even easier to scale up. Indeed, the performance of the code can be improved by increasing the number of qudit levels, thus making it possible to correct higher-order dephasing errors. This can be done by considering larger nuclear spins, such as ${ }^{173} \mathrm{Yb}$ in $\mathrm{Yb}(\text { trensal })^{\sqrt{38}}(S=5 / 2)$ or ${ }^{51} \mathrm{~V}$ in $\operatorname{VOTPP}(S=7 / 2) \stackrel{50}{,}$ shown in Fig. 3 - (d,e). Some remarks are necessary here: although chemically easy, the extension of the Hilbert space must be combined with the design of suitable code words showing a large gain even at intermediate times ${ }^{52}$ or of shorter pulse sequences whose duration does not strongly increase with the number of levels. This requires that all addressed energy gaps are well separated in the spectrum (i.e. $\left|\omega_{i}-\omega_{j}\right|>\Omega_{\mathrm{R}}$ for significantly large $\Omega_{\mathrm{R}}$ ). This condition translates into significant real or effective quadrupole interactions ${ }^{39150}$ (in the case of a nuclear spin qudit) or zero-field splitting (for electronic spin systems) $\frac{34140}{3}$ Increasing the frequency separations allows decreasing the duration of the control pulses, which is fundamental to reduce the harmful effect of decoherence during the correction protocol.

A digital quantum simulator is a device able to efficiently mimic the dynamics of a quantum system different from the hardware ${ }^{54}$ This can be done by first mapping the target Hamiltonian onto the hardware and then decomposing the corresponding dynamics into a sequence of elementary one- and two-qubit gates, controlled by the experimenter, via the Suzuki-Trotter decomposition. 

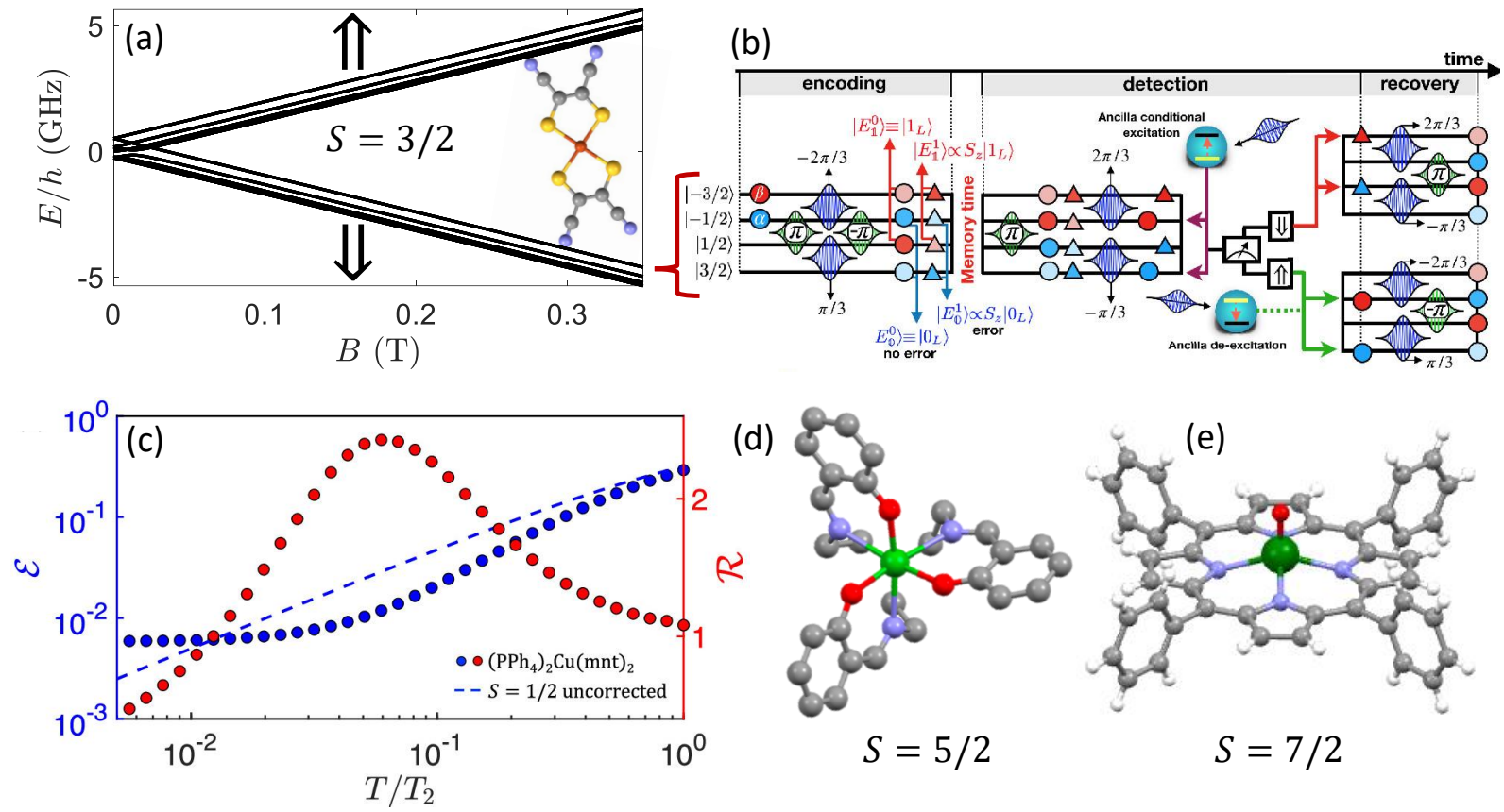

FIG. 3. Molecular spin qubits with embedded QEC. (a) Level diagram of $\left(\mathrm{PPh}_{4}\right)_{2}\left[\mathrm{Cu}(\mathrm{mnt})_{2}\right]$ (inset), embedding an $S=3 / 2$ nuclear spin ${ }^{21}$ hyperfine-coupled to an electronic spin $1 / 2$, as a function of the external field, applied along $z$ axis. Energy levels are split by the electronic Zeeman interaction into a low-lying electronic $\Downarrow$ subspace, in which the protected qubit is defined, and an excited electronic $\Uparrow$ manifold, with auxiliary levels needed for error detection. (b) Zoom on the electronic $\Downarrow$ subspace and corresponding sequence of radio-frequency/micro-wave pulses needed to correct an $S_{z}$ error on the qudit. Occurrence of this error brings each code word to an orthogonal state, thus making it possible to detect errors by a conditional excitation

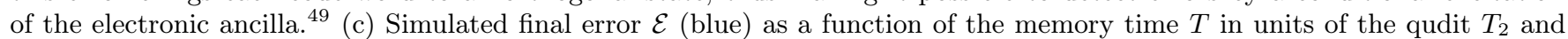
relative gain $\mathcal{R}=\mathcal{E}_{1 / 2} / \mathcal{E}$ (red) with respect to an isolated spin $1 / 2$ (characterized by error $\mathcal{E}_{1 / 2}$ ) with no correction. Thus, $\mathcal{R}$ measures the reduction of error obtained by QEC. (d,e) Other possible implementations using larger nuclear spin systems:

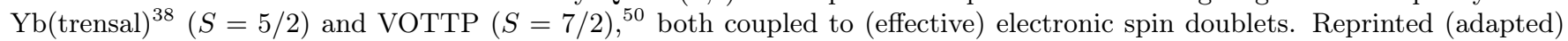
with permission from 49] Copyright American Chemical Society (2020).

The simplest quantum simulator based on molecular nanomagnets consists of a molecular chain of alternating spin $1 / 2$ qubits and different magnetic units acting as a switch for the qubit-qubit interaction. $32|56| 58$ The latter is effectively turned on by a conditional excitation of the switch, depending on the state of neighboring qubits via the qubit-switch coupling. This implements an entangling two-qubit controlled-phase gate, which, combined with single-qubit rotations, forms a universal set of gates and hence enables digital quantum simulation of a wide class of models, such as spin $\geq 1 / 2$ chains and fermionic systems. $56 \mid 59160$

Very recently, this idea has been extended to include units with $S>1 / 2 \cdot{ }^{61]}$ The multiple levels available within each qudit can simplify quantum simulation of models involving several degrees of freedom, such as bosonic fields interacting with matter ${ }^{61}$ The description of photon modes (including in principle an infinite number of levels) is a difficult task for qubit-based approaches, yielding an exponentially large Hilbert space or non-local interactions and thus deep quantum circuits $\sqrt[62]{64}$ Both the number of objects and the complexity of operations can be greatly simplified by pursuing a qudit-based approach, in which the photon space is truncated to the number of qudit levels.

The elementary unit of a molecular quantum simulator was realized in the following years, ${ }^{3215758}$ based on $\mathrm{Cr}_{7} \mathrm{Ni}$ molecular qubits with an interposed magnetic ion, in different geometries. This approach has however a possible limitation: scaling up this platform by simply extending the two-qubit units proposed in Refs. 32, 57, 58, and 65 can only be done up to about ten qubits, due to residual qubit-qubit couplings which are not completely suppressed by the scheme and become effective when elongating the register. Nevertheless, this idea can be combined with photon-induced coupling between different molecular processors to scale up (see next section).

A nuclear spin implementation of a molecule-based digital quantum simulator was also proposed, with the electronic spins mediating the coupling between qubits encoded in the nuclear spins of ${ }^{51} \mathrm{~V}$ ions ${ }^{59}$ This approach shows some remarkable advantages: first, nuclear spins are characterized by remarkably longer coherence, compared to their electronic counterpart. This benefit is usually canceled by slow nuclear qubit operations. In the scheme proposed in Ref. 59, the use of fast elec- 
tronic excitations makes two-qubit operations between nuclear spin qubits much faster than in standard NMR approaches, thus exploiting the long nuclear coherence. Second, large nuclear spins $\left(7 / 2\right.$ in the case of $\left.{ }^{51} \mathrm{~V}\right)$ paves the way to the use of qudits, embedding quantum error correction. A scheme for implementing two-qubit gates on such error protected nuclear spins has been recently put forward 51 and it could be extended in the near future to a more general class of gates and to quantum simulations.

\section{WIRING-UP MOLECULAR SPIN QUDITS}

While one could continue to increase the dimension of the molecular qudits, the progress in computational power will eventually be limited by serious technical difficulties. A major problem is associated with the "frequency crowding" of the required set of resonant transitions. This effect increasingly hinders addressing them spectroscopically, as it can already be seen in Fig. 2 (b) for $d=64$. There have proposals for introducing switchable couplers within molecular or supramolecular structures, formed by either ancillary spin qubits 58 or by molecular linkers that can modify its electronic structure under some external stimulus. ${ }^{66}$ Yet, at some point, this path must be complemented with the ability to locally control and, especially, wire up in a tunable manner different individual molecular spins.

An advantage of molecular nanomagnets in connection with this idea is that most of them are stable as individual units, e.g. in solution, and therefore can be transferred onto a solid substrate or a device. $67 \sqrt[69]{69}$ This has enabled the realization of spin-dependent electron transport experiments on single molecules. These experiments allow reading out and coherently controlling their nuclear $\underline{37 / 47}$ and electronic spin states $\frac{48}{4}$ and that have provided a first proof-of-principle realization of the Grover quantum search algorithm with a nuclear spin qudit!70

Here, we consider scaling up via the coupling of molecular spins to superconducting on-chip resonators ${ }^{46}$ The basic idea is to adapt to the realm of molecules techniques of circuit quantum electrodynamics, which was originally introduced as a platform for reading out and coherently communicating superconducting qubits ${ }^{71}$ The scheme is shown in Fig. 4. A number $N$ of magnetic molecules couple to the cavity mode of a resonator. Their spin states and energy levels can be controlled by a combination of global and local magnetic fields. When the coupling energy $G$ of each spin to a single photon is larger than the decoherence rates of the spin $1 / T_{2}$ (typically $>10^{4}$ $\mathrm{Hz}$ ) and of the cavity $\kappa$ (typically $<10^{4} \mathrm{~Hz}$ ), the shifts it induces on the resonance frequency $\Omega$ can be determined from the transmission through the device and it allows reading out the spin state. More importantly for our purpose here, it also introduces effective interactions between the spins! ${ }^{72}$ We have generalized this effective photon-mediated interactions to the case of spin qudits having multiple levels. The effective interaction Hamiltonian between two spin qudits is

$$
\mathcal{H}_{J}=\Omega \sum_{\vec{\alpha}, \vec{\beta}=1}^{2 S+1} \lambda_{1}^{\vec{\alpha}} \lambda_{2}^{\vec{\beta}}\left(\frac{1}{E_{\vec{\beta}}^{2}-\Omega^{2}}+\frac{1}{E_{\vec{\alpha}}^{2}-\Omega^{2}}\right) X_{1}^{\vec{\alpha}} X_{2}^{\vec{\beta}}
$$

where $\vec{\alpha} \equiv\left(\alpha_{1}, \alpha_{2}\right)$ denotes two eigenstates of one spin, separated by an energy gap $E_{\vec{\alpha}} \equiv E_{\alpha_{1}}-E_{\alpha_{2}}$ and connected by the Hubbard operator $X_{1}^{\alpha_{1}, \alpha_{2}} \equiv\left|\alpha_{1}\right\rangle\left\langle\alpha_{2}\right|$ (and similarly for $\vec{\beta}$ in the other spin). Constants $\lambda_{1}^{\vec{\alpha}}$ and $\lambda_{2}^{\vec{\beta}}$ depend on the wave functions of these states and are proportional to the spin-photon coupling $G$. Equation (1) is derived in the dispersive regime, i.e. the spins' frequencies are non resonant with the cavity. Several architectures use such photon mediated couplings to generate two-qubit entangling gates $73 / 74$. In particular, it allows to swap states of two qudits, as illustrated in Fig. 4. Together with single-qudit operations reported in Fig. 2 , the coupling (1) ensures complete control of the two-qudit system, thus forming a universal set.

This scheme works if a sufficiently strong coupling $G$ is attained. For conventional coplanar superconducting resonators, $\stackrel{75}{75}$ the typical couplings amount to a few $\mathrm{Hz}$, way below even the best decoherence rates for molecular spins. In order to overcome this limitation, one needs to bridge the gap that separates the sizes of the circuit and the molecule in order to locally enhance $G$. A direct method is decreasing the width of the inductor down to a few nm. ${ }^{76 / 77}$ It was predicted theoretically, ${ }^{46 / 76}$ and recently confirmed by experiments, $\frac{78}{,}$ that squeezing in this manner the photon magnetic field can increase $G$ by several orders of magnitude (see Fig. 4). A complementary approach is to work with very low inductance $L C$ lumped-element resonators, which show large current densities at resonance. ${ }^{79}$ It is expected ${ }^{78}$ that the combination of both approaches can take $G$ for single spins close to tenths of $\mathrm{MHz}$. This would suffice to reach the coherent coupling regime for single molecular spin qudits provided that $T_{2}>10 \mu \mathrm{s}$, which seems feasible.

\section{OUTLOOK AND CONCLUSIONS}

The previous sections show that artificial magnetic molecules can contribute to reach higher levels of computational power along two complementary directions: reducing the computational costs of some algorithms and providing new methods for wiring up additional quantum resources. A paradigmatic example is QEC. Encoding a protected qubit in a single physical object can greatly simplify the practical implementation of both error correction and the quantum logic on the pro-

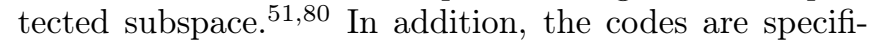
cally adapted to the energy level schemes of the molecular spin qudits and to their dominant error sources. Even more, they can be optimized in several ways. First, by carefully evaluating the interactions with nuclear spins 

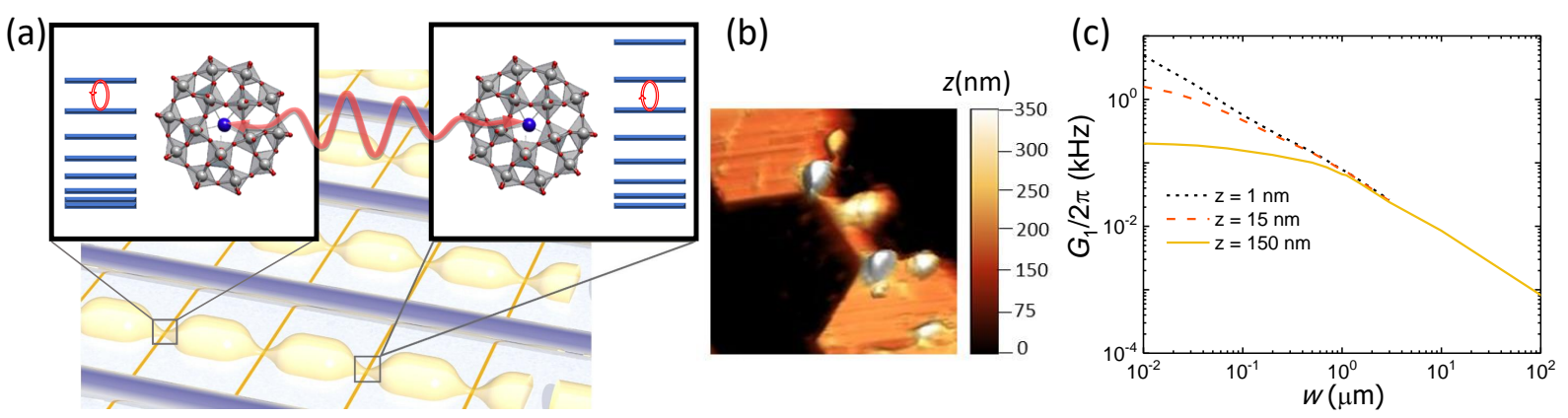

FIG. 4. (a) Scheme for wiring up molecular spin qudits through their coupling to a common cavity mode in a superconducting resonator. The photon mediated interaction between suitably tuned qudits allows the implementation of two-qudit gates and ensures universal operation. This scheme allows different levels of scalability: using multiple levels inside each qudit, wiring up several of them in a resonator and coupling different resonators on a chip. (b) Atomic Force Microscopy (AFM) image of a nanoconstriction fabricated in the central line of a superconducting resonator and of nanodrops of DPPH $S=1 / 2$ free radical molecules deposited onto it by the same AFM tip. (c) Dependence of the single spin (for $S=1 / 2$ ) to single photon coupling as a function of the central line width $w$ and of the distance $z$ between the molecule and the constriction.

in the molecules and their effect of the qudit states. Second, by optimizing the pulse sequences used in the protocols. Similar considerations apply to the implementation of some quantum simulations, which benefit from the multiple level structure that is inherent to the qudit and from the simplification associated with the avoidance of non-local operations.

This approach has already led to a first proof-ofconcept implementation of Grover's search algorithm on a $d=3$ spin qudit. ${ }^{70}$ We foresee that more will follow in the next few years, increasing the complexity of the computational Hilbert space to $3-4$ qubits (or $d=8-16$ ), enough to realize the simplest QEC codes and quantum simulations. An advantage of several quantum simulation and computation algorithms realized on a molecular architecture is that they do not necessarily require measuring the response of single molecules. Depending on the spin states involved, they can be performed by using broad-band ESR or NMR experiments on magnetically diluted crystals of molecular qudits. For this reason, probably the best suited candidates are qudits based on internal spin states of individual ions (either electronic, nuclear or a combination of both). The main technical requisite is to attain sufficiently long $T_{2}>10-20 \mu \mathrm{s}$ in these crystals, which is still quite demanding but seems within reach for sufficiently low spin concentrations and adequately designed ligands. A promising alternative is the use of on-chip resonators coupled to transmission lines, 3918184 which can help to widen the frequency ranges for the excitation and read-out as well as to attain stronger microwave magnetic fields, thus faster operation rates.

Reaching the next level in scalability necessarily involves a modular approach and the ability of coherently exchanging information between different spins. Progress along these lines will probably rely on a combination of bottom-up strategies with solid-state circuits fabricated by top-down lithography. We have shown in section IV that the coherent coupling to on-chip superconducting resonators provides a scalable path to wire up and perform universal operations with several molecular spin qudits. In principle, this route can give rise to processors hosting several tens of qudits, each of them acting as a qubit with embedded error protection. Besides, different resonators can be integrated in a chip using technologies developed for platforms based on superconducting circuits. Achieving the strong coupling of individual molecular processors with a resonator would also enable projective measurements of the molecular state. In contrast to NMR approaches, this makes it possible to initialize the register also by measuring. Moreover, it greatly enlarges the range of possible algorithms.

Yet, attaining $G T_{2}>1$ for single spins is still very challenging, even though it appears feasible in a medium term. Enhancing the spin-photon coupling will probably require the combination of optimally designed resonators and a local confinement of photons near superconducting nano-structures. Electric fields can be confined much more easily than magnetic fields. Therefore, a way to improve on the latter aspect is to use molecules showing a large spin-electric coupling. ${ }^{85} \mathrm{~A}$ different alternative is to exploit a resonant spin-photon coupling to exchange information between spins. ${ }^{86}$ Yet another one is to use the spin-magnon coupling. The latter would play the role of microwave photons in the superconducting resonators or cavities. Strong coupling between spins and the Kittel mode in a YIG nanosphere has been proposed ${ }^{[87}$ The formalism advocated here can easily be exported to these new architectures. Work on the devices must be accompanied by progress in the deposition of few molecules with an exquisite control over the position and the proper interface with the circuit surface. An option is to profit from either self-organization or synthesis of molecular arrays at the surface ${ }^{88}$ From the molecular design, it is important to maximize the transition matrix elements that determine the value of $G$, e.g. by using low-lying levels 
of high-spin molecules to define the qudit states. ${ }^{76}$

We finally note that work along this direction has also a significant impact on very important applications beyond the realm of quantum computing. Increasing the coupling of superconducting resonators to spins contributes to the development of on-chip magnetic resonance with a sensitivity able of detecting samples of $\sim 10^{-2}$ pico$\mathrm{L}$, or even magnetic excitations of individual magnetic

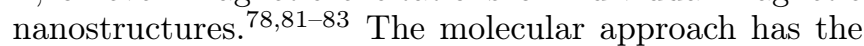
added value of serving as a suitable vehicle to deliver diverse samples, as well as to improve their interface with the circuit. Finally, these systems are also ideal for exploring the quantum electrodynamical control of matter ${ }^{89}$ and, in particular, for modifying and controlling long-range magnetically ordered phases $[90$

In summary, even though unleashing the full potential of this hybrid technology, i.e. creating large-scale molecular spin-based processors, will likely require further developments across different disciplines, the demonstration of its key ingredients, namely the operation over molecular scale NISQs and the ability to coherently couple two of them, seem well within the reach of current technologies.

The data that support the findings of this study are available from the corresponding author upon reasonable request.

\section{ACKNOWLEDGMENTS}

We thank J Román-Roche and O. Roubeau for helping us with Figures 2 and 4 respectively. We acknowledge funding from the European Union's Horizon 2020 research and innovation programme (QUANTERA project SUMO, FET-OPEN grant 862893 FATMOLS), the Spanish MICINN (grants RTI2018-096075-B-C21, PCI2018-093116, PGC2018-094792-B-I00), the Gobierno de Aragón (grant E09-17R-Q-MAD), the CSIC Quantum Technology Platform PT-001, and the Italian Ministry of Education and Research (MIUR) through the co-funding of SUMO.

${ }^{1}$ E. Gibney, "Physics: Quantum computer quest," Nature 516, 24-26 (2014)

${ }^{2}$ M. Mohseni, P. Read, H. Neven, S. Boixo, V. Denchev, R. Babbush, A. Fowler, V. Smelyanskiy, and J. Martinis, "Commercialize quantum technologies in five years," Nature 543, 171-175 (2017)

${ }^{3}$ A. G. Fowler, M. Mariantoni, J. M. Martinis, and A. N. Cleland, "Surface codes: Towards practical large-scale quantum computation," Phys. Rev. A 86, 032324 (2012)

${ }^{4} \mathrm{E}$. Knill and R. Laflamme, "Theory of quantum error-correcting codes," Phys. Rev. A 55, 900-911 (1997).

${ }^{5}$ B. M. Terhal, "Quantum error correction for quantum memories," Rev. Mod. Phys. 87, 307-346 (2015)

${ }^{6}$ J. Preskill, "Quantum computing in the nisq era and beyond," Quantum 2, 79 (2018)

${ }^{7} \mathrm{~F}$. Arute and et al, "Quantum supremacy using a programmable superconducting processor," Nature 574, 505--510 (2019)

${ }^{8}$ A. W. Cross, L. S. Bishop, S. Sheldon, P. D. Nation, and J. M. Gambetta, "Validating quantum computers using randomized model circuits," Phys. Rev. A 100, 032328 (2019)
${ }^{9}$ D. Loss and D. P. DiVincenzo, "Quantum computation with quantum dots," Phys. Rev. A 57, 120-126 (1998)

${ }^{10}$ J. J. Pla, K. Y. Tan, J. P. Dehollain, W. H. Lim, J. J. L. Morton, D. N. Jamieson, A. S. Dzurak, and A. Morello, "A single-atom electron spin qubit in silicon," Nature 489, 541-545 (2012)

${ }^{11}$ J. J. L. Morton, D. R. McCamey, M. A. Eriksson, and S. A. Lyon, "Embracing the quantum limit in silicon computing," $\mathrm{Na}$ ture 479, 345-353 (2011)

${ }^{12}$ D. D. Awschalom, L. C. Bassett, A. S. Dzurak, E. L. Hu, and J. R. Petta, "Quantum spintronics: Engineering and manipulating atom-like spins in semiconductors," Science 339, 1174-1179 (2013)

${ }^{13}$ N. W. Hendrickx, W. I. L. Lawrie, M. Russ, F. v. Riggelen, S. L. de Snoo, R. N. Schouten, A. Sammak, G. Scappucci, and M. Veldhorst, "A four-qubit germanium quantum processor," $\mathrm{Na}-$ ture 591, 580--585 (2021)

${ }^{14}$ A. Ardavan, O. Rival, J. J. L. Morton, S. J. Blundell, A. M. Tyryshkin, G. A. Timco, and R. E. P. Winpenny, "Will SpinRelaxation Times in Molecular Magnets Permit Quantum Information Processing?" Phys. Rev. Lett. 98, 057201 (2007)

${ }^{15}$ J. M. Zadrozny, J. Niklas, O. G. Poluektov, and D. E. Freedman, "Millisecond coherence time in a tunable molecular electronic spin qubit." ACS Cent. Sci. 1, 488 (2015).

${ }^{16}$ M. Shiddiq, D. Komijani, Y. Duan, A. Gaita-Ariño, E. Coronado, and S. Hill, "Enhancing coherence in molecular spin qubits via atomic clock transitions." Nature 531, 348-351 (2016).

${ }^{17}$ A. Gaita-Ariño, F. Luis, S. Hill, and E. Coronado, "Molecular spins for quantum computation." Nature Chem. 11, 301-309 (2019).

${ }^{18}$ M. Atzori and R. Sessoli, "The second quantum revolution: Role and challenges of molecular chemistry," J. Am. Chem. Soc. 141, 11339 (2019).

${ }^{19}$ C. J. Wedge, G. A. Timco, E. T. Spielberg, R. E. George, F. Tuna, S. Rigby, E. J. L. McInnes, R. E. P.Winpenny, S. J. Blundell, and A. Ardavan, "Chemical engineering of molecular qubits." Phys. Rev. Lett. 108, 107204 (2012).

${ }^{20}$ M. J. Martínez-Pérez, S. Cardona-Serra, C. Schlegel, F. Moro, P. J. Alonso, H. Prima-García, J. M. Clemente-Juan, M. Evangelisti, A. Gaita-Ariño, J. Sesé, J. van Slageren, E. Coronado, and F. Luis, "Gd-based single-ion magnets with tunable magnetic anisotropy: Molecular design of spin qubits," Phys. Rev. Lett. 108, 247213 (2012)

${ }^{21} \mathrm{~K}$. Bader, D. Dengler, S. Lenz, B. Endeward, S.-D. Jiang, P. Neugebauer, and J. van Slageren, "Room temperature quantum coherence in a potential molecular qubit," Nat. Commun 5, $5304(2014)$.

${ }^{22}$ C.-J. Yu, M. J. Graham, J. M. Zadrozny, J. Niklas, M. D. Krzyaniak, M. R. Wasielewski, O. G. Poluektov, and D. E. Freedman, "Long coherence times in nuclear spin-free vanadyl qubits," J. Am. Chem. Soc. 138, 14678-14685 (2016).

${ }^{23}$ M. Atzori, E. Morra, L. Tesi, A. Albino, M. Chiesa, L. Sorace, and R. Sessoli, "Quantum coherence times enhancement in vanadium(iv)-based potential molecular qubits: the key role of the vanadyl moiety," J. Am. Chem. Soc. 138, 11234-11244 (2016).

${ }^{24}$ J. M. Zadrozny, A. T. Gallagher, T. D. Harris, and D. E. Freedman, "A porous array of clock qubits," J. Am. Chem. Soc. 139, $7089-7094$ (2017).

${ }^{25}$ C. A. Collett, K.-I. Ellers, N. Russo, K. R. Kittilstved, G. A. Timco, R. E. P. Winpenny, and J. R. Friedman, "A clock transition in the $\mathrm{cr}_{7} \mathrm{mn}$ molecular nanomagnet," Magnetochemistry 5, 4 (2019).

${ }^{26}$ M. Rubín-Osanz, F. Lambert, F. Shao, E. Rivière, R. Guillot, N. Suaud, N. Guihéry, D. Zueco, A.-L. Barra, T. Mallah, and F. Luis, "Chemical tuning of spin clock transitions in molecular monomers based on nuclear spin-free ni(ii)," Chem. Sci. 12, 5123$5133(2021)$

${ }^{27}$ G. Aromí, D. Aguilà, F. Luis, S. Hill, and E. Coronado, "Design of magnetic coordination complexes for quantum computing." Chem. Soc. Rev. 41, 537-546 (2012). 
${ }^{28}$ F. Luis, A. Repollés, M. J. Martínez-Pérez, D. Aguilà, O. Roubeau, D. Zueco, P. J. Alonso, M. Evangelisti, A. Camón, J. Sesé, L. A. Barrios, and G. Aromí, "Molecular prototypes for spin-based cnot and swap quantum gates." Phys. Rev. Lett. 107, 117203 (2011).

${ }^{29}$ D. Aguilà, D. Barrios, V. Velasco, O. Roubeau, A. Repollés, P. Alonso, J. Sesé, S. Teat, F. Luis, and G. Aromí, "Heterodimetallic [lnln'] lanthanide complexes: Toward a chemical design of two-qubit molecular spin quantum gates." J. Am. Chem. Soc. 136, 14215 (2014).

${ }^{30}$ E. Macaluso, M. Rubín, D. Aguilà, A. Chiesa, J. I. M. L. A. Barrios, P. J. Alonso, O. Roubeau, F. Luis, G. Aromí, and S. Carretta, "A heterometallic [lnln'ln] lanthanide complex as a qubit with embedded quantum error correction," Chem. Sci. 11, 10337 (2020).

${ }^{31}$ A. Ardavan, A. M. Bowen, A. Fernandez, A. J. Fielding, D. Kaminski, F. Moro, C. A. Muryn, M. D.Wise, A. Ruggi, E. J. L. McInnes, K. Severin, C. R. T. G. A. Timco, F. Tuna, G. F. S. Whitehead, and R. E. P.Winpenny, "Engineering coherent interactions in molecular nanomagnet dimers." npj Quantum Information 1, 15012 (2015).

${ }^{32}$ J. Ferrando-Soria, E. Moreno-Pineda, A. Chiesa, A. Fernández, S. A. Magee, S. Carretta, P. Santini, I. Vitorica-Yrezabal, F. Tuna, E. J. L. McInness, and R. E. P. Winpenny, "A modular design of molecular qubits to implement universal quantum gates." Nat. Commun. 7, 11377 (2016).

${ }^{33}$ A. Fernández, J. Ferrando-Soria, E. Moreno-Pineda, F. Tuna, I. n. J. Vitorica-Yrezabal, C. Knappke, J. Ujma, C. A. Muryn, G. A. Timco, P. E. Barran, A. Ardavan, and R. E. Winpenny, "Making hybrid [n]-rotaxanes as supramolecular arrays of molecular electron spin qubits." Nat. Commun. 7, 10240 (2016).

${ }^{34}$ M. D. Jenkins, Y. Duan, B. Diosdado, J. J. García-Ripoll, A. Gaita-Ariño, C. Giménez-Saiz, P. J. Alonso, E. Coronado, and F. Luis, "Coherent manipulation of three-qubit states in a molecular single-ion magnet," Phys. Rev. B 95, 064423 (2017).

${ }^{35}$ E. Moreno-Pineda, M. Damjanović, O. Fuhr, W. Wernsdorfer, and M. Ruben, "Nuclear spin isomers: Engineering a et4n[dypc2] spin qudit," Angewandte Chemie International Edition 56, 99159919 (2017)

${ }^{36}$ E. Moreno-Pineda, C. Godfrin, F. Balestro, W. Wernsdorfer, and M. Ruben, "Molecular spin qudits for quantum algorithms," Chem. Soc. Rev. 47, 501-513 (2018)

${ }^{37}$ S. Thiele, F. Balestro, R. Ballou, S. Klyatskaya, M. Ruben, and W. Wernsdorfer, "Electrically driven nuclear spin resonance in single-molecule magnets," Science 344, 1135-1138 (2014).

${ }^{38}$ R. Hussain, G. Allodi, A. Chiesa, E. Garlatti, D. Mitcov, A. Konstantatos, K. S. Pedersen, R. De Renzi, S. Piligkos, and S. Carretta, "Coherent manipulation of a molecular ln-based nuclear qudit coupled to an electron qubit," Journal of the American Chemical Society 140, 9814-9818 (2018)

${ }^{39}$ I. Gimeno, A. Urtizberea, J. Román-Roche, D. Zueco, A. Camón, P. J. Alonso, O. Roubeau, and F. Luis, "Broad-band spectroscopy of a vanadyl porphyrin: a model electronuclear spin qudit," Chem. Sci. 12, 5621-5630 (2021)

${ }^{40}$ F. Luis, P. J. Alonso, O. Roubeau, V. Velasco, D. Zueco, D. Aguilà, J. I. Martínez, L. A. Barrios, and G. Aromí, "A dissymmetric $\left[\mathrm{gd}_{2}\right]$ coordination molecular dimer hosting six addressable spin qubits," Communications Chemistry 3, 176 (2020)

${ }^{41}$ D. P. DiVincenzo, "The physical implementation of quantum computation," Fortschritte der Physik 48, 771 (2000)

${ }^{42}$ L. M. K. Vandersypen and I. L. Chuang, "Nmr techniques for quantum control and computation," Rev. Mod. Phys. 76, 10371069 (2005)

${ }^{43}$ A. Muthukrishnan and C. R. Stroud, "Multivalued logic gates for quantum computation," Phys. Rev. A 62, 052309 (2000)

${ }^{44}$ G. K. Brennen, D. P. O'Leary, and S. S. Bullock, "Criteria for exact qudit universality," Phys. Rev. A 71, 052318 (2005)

${ }^{45}$ C. V. Kraus, M. M. Wolf, and J. I. Cirac, "Quantum simulations under translational symmetry," Phys. Rev. A 75, 022303 (2007)

${ }^{46}$ M. D. Jenkins, D. Zueco, O. Roubeau, G. Aromí, J. Majer, and
F. Luis, "A scalable architecture for quantum computation with molecular nanomagnets." Dalton Trans. 45, 16682 (2016).

${ }^{47}$ R. Vincent, S. Klyatskaya, M. Ruben, W. Wernsdorfer, and F. Balestro, "Electronic read-out of a single nuclear spin using a molecular spin transistor," Nature 488, 357-360 (2012)

${ }^{48}$ C. Godfrin, S. Thiele, A. Ferhat, S. Klyatskaya, M. Ruben, W. Wernsdorfer, and F. Balestro, "Electrical read-out of a single spin using an exchange-coupled quantum dot," Nano Lett. 11, 3984-3989 (2017)

${ }^{49}$ A. Chiesa, E. Macaluso, F. Petiziol, S. Wimberger, P. Santini, and S. Carretta, "Molecular nanomagnets as qubits with embedded quantum-error correction," The Journal of Physical Chemistry Letters 11, 8610-8615 (2020)

${ }^{5}$ S. Chicco, A. Chiesa, G. Allodi, E. Garlatti, M. Atzori, L. Sorace, R. De Renzi, R. Sessoli, and S. Carretta, "Controlled coherent dynamics of [vo(tpp)], a prototype molecular nuclear qudit with an electronic ancilla," submitted (2021).

${ }^{51}$ A. Chiesa, F. Petiziol, E. Macaluso, S. Wimberger, P. Santini, and S. Carretta, "Embedded quantum-error correction and controlled-phase gate for molecular spin qubits," AIP Advances 11, 025134 (2021)

${ }^{52}$ F. Petiziol, A. Chiesa, S. Wimberger, P. Santini, and S. Carretta, "Counteracting dephasing in molecular nanomagnets by optimised qudit encodings," submitted (2021).

${ }^{53}$ C. Cafaro, F. Maiolini, and S. Mancini, "Quantum stabilizer codes embedding qubits into qudits," Phys. Rev. A 86, 022308 (2012)

${ }^{54}$ F. Tacchino, A. Chiesa, S. Carretta, and D. Gerace, "Quantum computers as universal quantum simulators: state-of-art and perspectives." Adv. Quantum Technol. , 1900052 (2019).

${ }^{55}$ S. Lloyd, "Universal quantum simulators," Science 273, 10731078 (1996)

${ }^{56}$ P. Santini, S. Carretta, F. Troiani, and G. Amoretti, "Molecular nanomagnets as quantum simulators," Phys. Rev. Lett. 107, $230502(2011)$

${ }^{\prime}$ A. Chiesa, G. F. S. Whitehead, S. Carretta, L. Carthy, G. A. Timco, S. J. Teat, G. Amoretti, E. Pavarini, R. E. P. Winpenny, and P. Santini, "Molecular nanomagnets with switchable coupling for quantum simulation." Sci. Rep. 4, 7423 (2014).

${ }^{58}$ e. a. J. Ferrando-Soria, "Switchable interactions in molecular double qubits." Chem 1, 727-752 (2016).

${ }^{59}$ M. Atzori, A. Chiesa, E. Morra, M. Chiesa, L. Sorace, S. Carretta, and R. Sessoli, "A two-qubit molecular architecture for electron mediated nuclear quantum simulation." Chem. Sci. 9, $6183(2018)$.

${ }^{60}$ A. Chiesa, P. Santini, D. Gerace, J. Raftery, A. A. Houck, and S. Carretta, "Digital quantum simulators in a scalable architecture of hybrid spin-photon qubits." Sci. Rep. 5, 16036 (2015).

${ }^{61}$ F. Tacchino, A. Chiesa, R. Sessoli, I. Tavernelli, and S. Carretta, "Molecular spin qudits for quantum simulation of light-matter interactions," submitted (2021).

${ }^{62}$ N. P. D. Sawaya, T. Menke, T. H. Kyaw, S. Johri, A. AspuruGuzik, and G. G. Guerreschi, "Resource-efficient digital quantum simulation of d-level systems for photonic, vibrational, and spin-s Hamiltonians," npj Quantum Information 6, 49 (2020)

${ }^{63}$ S. V. Mathis, G. Mazzola, and I. Tavernelli, "Toward scalable simulations of lattice gauge theories on quantum computers," Phys. Rev. D 102, 094501 (2020).

${ }^{64}$ A. DiPaolo, P. K. Barkoutsos, I. Tavernelli, and A. Blais, "Variational quantum simulation of ultrastrong light-matter coupling." Phys. Rev. Research 2, 033364 (2020).

${ }^{65}$ A. Chiesa, P. Santini, and S. Carretta, "Supramolecular complexes for quantum simulation," Magnetochemistry 2 (2016), 10.3390/magnetochemistry2040037

${ }^{{ }^{\circ}}$ J. S. Uber, M. Estrader, J. García, P. Lloyd-Williams, A. Sadurní, D. Dengler, J. van Slageren, N. F. Chilton, O. Roubeau, S. J. Teat, J. Ribas-Ariño, and G. Aromí, "Molecules designed to contain two weakly coupled spins with a photoswitchable spacer," Chemistry - A European Journal 23, 13648-13659 (2017) 
${ }^{67}$ M. Mannini, F. Pineider, C. Danieli, F. Totti, L. Sorace, P. Sainctavit, M.-A. Arrio, E. Otero, L. Joly, J. C. Cezar, A. Cornia, and R. Sessoli, "Quantum tunnelling of the magnetization in a monolayer of oriented single-molecule magnets," Nature 468, 417-421 (2010)

${ }^{68}$ N. Domingo, E. Bellido, and D. Ruiz-Molina, "Advances on structuring, integration and magnetic characterization of molecular nanomagnets on surfaces and devices," Chem. Soc. Rev. 41, 258-302 (2012)

${ }^{{ }^{69}}$ L. Malavolti, M. Briganti, M. Hänze, G. Serrano, I. Cimatti, G. McMurtrie, E. Otero, P. Ohresser, F. Totti, M. Mannini, R. Sessoli, and S. Loth, "Tunable Spin-Superconductor Coupling of Spin 1/2 Vanadyl Phthalocyanine Molecules," Nano Lett. 18, $7955(2018)$

${ }^{\mathrm{T}} \mathrm{C}$. Godfrin, A. Ferhat, R. Ballou, S. Klyatskaya, M. Ruben, W. Wernsdorfer, and F. Balestro, "Operating quantum states in single magnetic molecules: Implementation of grover's quantum algorithm." Phys. Rev. Lett. 119, 187702 (2017).

${ }^{71}$ A. Blais, R.-S. Huang, A. Wallraff, S. M. Girvin, and R. J. Schoelkopf, "Cavity quantum electrodynamics for superconducting electrical circuits: An architecture for quantum computation," Phys. Rev. A 69, 062320 (2004)

${ }^{72}$ D. Zueco, G. M. Reuther, S. Kohler, and P. Hänggi, "Qubitoscillator dynamics in the dispersive regime: Analytical theory beyond the rotating-wave approximation," Phys. Rev. A 80, $033846(2009)$

${ }^{73}$ C. Rigetti and M. Devoret, "Fully microwave-tunable universal gates in superconducting qubits with linear couplings and fixed transition frequencies," Physical Review B 81 (2010), 10.1103/physrevb.81.134507

${ }^{4}$ J. M. Chow, A. D. Córcoles, J. M. Gambetta, C. Rigetti, B. R. Johnson, J. A. Smolin, J. R. Rozen, G. A. Keefe, M. B. Rothwell, M. B. Ketchen, and M. Steffen, "Simple all-microwave entangling gate for fixed-frequency superconducting qubits," Physical Review Letters 107 (2011), 10.1103/physrevlett.107.080502.

${ }^{15}$ A. Wallraff, D. I. Schuster, A. Blais, L. Frunzio, R.-S. Huang, J. Majer, S. Kumar, S. M. Girvin, and R. J. Schoelkopf, "Strong coupling of a single photon to a superconducting qubit using circuit quantum electrodynamics," Nature 431, 162-167 (2004)

${ }^{76}$ M. D. Jenkins, T. Hümmer, M. J. Martínez-Pérez, J. GarcíaRipoll, D. Zueco, and F. Luis, "Coupling single-molecule magnets to quantum circuits," New J. Phys. 15, 095007 (2013)

${ }^{77}$ M. D. Jenkins, U. Naether, M. Ciria, J. Sesé, J. Atkinson, C. Sánchez-Azqueta, E. d. Barco, J. Majer, D. Zueco, and F. Luis, "Nanoscale constrictions in superconducting coplanar waveguide resonators," Appl. Phys. Lett. 105, 162601 (2014)

${ }^{78}$ I. Gimeno, W. Kersten, M. C. Pallarés, P. Hermosilla, M. J. Martínez-Pérez, M. D. Jenkins, A. Angerer, C. Sánchez-Azqueta, D. Zueco, J. Majer, A. Lostao, and F. Luis, "Enhanced Molecular Spin-Photon Coupling at Superconducting Nanoconstrictions,"
ACS Nano 14, 8707-8715 (2020)

${ }^{79}$ B. Sarabi, P. Huang, and N. M. Zimmerman, "Possible Hundredfold Enhancement in the Direct Magnetic Coupling of a SingleAtom Electron Spin to a Circuit Resonator," Phys. Rev. Applied 11, 014001 (2019)

${ }^{80}$ L. Hu, Y. Ma, W. Cai, X. Mu, Y. Xu, W. Wang, Y. Wu, H. Wang, Y. P. Song, C.-L. Zou, S. M. Girvin, L.-M. Duan, and L. Sun, "Quantum error correction and universal gate set operation on a binomial bosonic logical qubit," Nat. Phys. 15, 503-508 (2019)

${ }^{81}$ A. Bienfait, A. A. Pla, Y. Kubo, X. Stern, M. Zhou, C. C. Lo, C. D. Weis, T. Schenkel, M. L. W. Thewalt, D. Vion, D. Esteve, B. Julsgaard, K. Mølmer, J. J. L. Morton, and P. Bertet, "Reaching the quantum limit of sensitivity in electron spin resonance," Nature Nanotech. 11, 253-257 (2016)

${ }^{82}$ C. Eichler, A. J. Sigillito, S. A. Lyon, and J. R. Petta, "Electron Spin Resonance at the Level of $10^{4}$ Spins Using Low Impedance Superconducting Resonators," Phys. Rev. Lett. 118, 037701 (2017)

${ }^{83}$ S. Probst, A. Bienfait, P. Campagne-Ibarcq, J. J. Pla, B. Albanese, J. F. Da Silva Barbosa, T. Schenkel, D. Vion, D. Esteve, K. Mølmer, J. J. L. Morton, R. Heeres, and P. Bertet, "Inductivedetection electron-spin resonance spectroscopy with 65 spins / $\mathrm{Hz}$ sensitivity," Appl. Phys. Lett. 111, 202604 (2017)

${ }^{84}$ C. Bonizzoni, A. Ghirri, F. Santanni, M. Atzori, L. Sorace, R. Sessoli, and M. Affronte, "Storage and retrieval of microwave pulses with molecular spin ensembles," npj Quantum Information 6, $68(2020)$

${ }^{8}$ J. Liu, J. Mrozek, W. K. Myers, G. A. Timco, R. E. P. Winpenny, B. Kintzel, W. Plass, and A. Ardavan, "Electric field control of spins in molecular magnets," Phys. Rev. Lett. 122, 037202 (2019)

${ }^{86}$ S. Carretta, A. Chiesa, F. Troiani, D. Gerace, G. Amoretti, and P. Santini, "Quantum information processing with hybrid spinphoton qubit encoding," Phys. Rev. Lett. 111, 110501 (2013)

${ }^{87}$ T. Neuman, D. S. Wang, and P. Narang, "Nanomagnonic cavities for strong spin-magnon coupling and magnon-mediated spin-spin interactions," Physical Review Letters 125 (2020), 10.1103/physrevlett.125.247702

${ }^{88}$ A. Urtizberea, E. Natividad, P. J. Alonso, L. Pérez-Martínez, M. A. Andrés, I. Gascón, I. Gimeno, F. Luis, and O. Roubeau, "Vanadyl spin qubit 2d arrays and their integration on superconducting resonators," Mater. Horiz. 7, 885-897 (2020)

${ }^{89} \mathrm{M}$. Ruggenthaler, N. Tancogne-Dejean, J. Flick, H. Appel, and A. Rubio, "From a quantum-electrodynamical light-matter description to novel spectroscopies," Nature Reviews Chemistry 2 (2018), 10.1038/s41570-018-0118.

${ }^{90}$ J. Román-Roche, F. Luis, and D. Zueco, "Photon condensation in magnetic cavity qed," arXiv preprint arXiv:2011.03753 (2020). 